\title{
Facial measurements in the newborn (towards syndrome delineation)
}

\author{
O O Omotade
}

\begin{abstract}
Inner and outer canthal distances, palpebral fissure length, occipitofrontal circumference, and canthal index values were determined and compared between 252 white newborns in Cardiff and 256 black newborns in Ibadan, Nigeria. The study showed that the white and black newborns had the same inner canthal distance while the outer canthal distance and palpebral fissure length were significantly smaller in the white newborns than in their black counterparts. A simple instrument was also constructed and advocated for use. It is concluded that for facial measurements local values should be used in the evaluation of dysmorphic states.
\end{abstract}

It is no longer acceptable to describe dysmorphic physical findings without using proper measurements. Claims such as low set ears and widely spaced eyes and nipples should not be made unless they are accompanied by accurate measurements. ${ }^{1}$

Normal values for facial measurements have been recorded for many communities. Laestadius, ${ }^{2}$ Feingold and Gellis, ${ }^{3}$ and Lucas and Pryor ${ }^{4}$ recorded normal facial measurements for the white population. Juberg $e t a l^{5}$ recorded the intercanthal distances of 5 to 11 year old American blacks. Some values were established by Ogunye $e t a l^{6}$ and Ejiwunmi $e t a l^{7}$ for the newborn Nigerian Yoruba population.

However, these workers did not measure the same facial parameters, for example, Ogunye et $a^{6}{ }^{6}$ measured the outer orbital distance while Ejiwunmi et $a l^{7}$ measured the outer canthal distance. These two measurements differ and there cannot be any basis for comparison. Apart from Feingold and Gellis, ${ }^{3}$ who compared a relatively small number of blacks with their Caucasian counterparts, no other workers have compared Negroid and Caucasoid subjects in the same study using the same equipment.

Institute of Child Health, College of Medicine, University College Hospital, Ibadan, Nigeria.

O O Omotade

Received for publication 27 June 1989.

Revised version accepted for publication 20 December 1989.
It is proposed in this study to (1) present normal values for facial measurements in newborns; (2) to compare these values between whites and blacks; and (3) present a simple piece of equipment for facial measurement.

\section{Materials and methods}

The subjects comprised two groups of normal term neonates, 252 white newborns in Cardiff, Wales and 256 black newborns in Ibadan, Nigeria. The Cardiff babies were delivered at the maternity unit of the University Hospital of Wales and St David's Hospital. These two hospitals were the only centres for hospital delivery in Cardiff. The Ibadan babies were chosen from those delivered at the University College Hospital (UCH) and Adeoyo State Hospital (ASH). These two hospitals are the largest in Ibadan and deliver the majority of hospital births within the city. All the babies were examined first and only those declared normal were included in the study. A 'normal' neonate was one who had no congenital abnormality or had only one minor abnormality such as a preauricular sinus. ${ }^{8}$

The gestational age was determined using the first day of the last menstrual period in addition to ultrasound scanning for biparietal diameter estimation in the Cardiff group, and from the first day of the last menstrual period and the Dubowitz scoring system $^{9}$ in the Ibadan group. All measurements were taken within 72 hours of birth.

The facial measurements were taken with an instrument constructed from exposed $x$ ray film. A vertical line was drawn passing through the middle of the film. This line intersected another horizontal line at right angles in the middle of the film, thereby dividing it into four quadrants. The horizontal line was then marked into millimetres. At $2 \mathrm{~mm}$ apart, vertical lines were drawn from the top to the bottom, separating the horizontal line into $2 \mathrm{~mm}$ divisions. This film was then photographed and developed negatively. The originally bluish colouration then became transparent and permanent. This is a modification of the instrument used by Feingold $e t a l^{3}$ for ear measurement.

The facial measurements were determined by holding the transparency horizontally above and close 


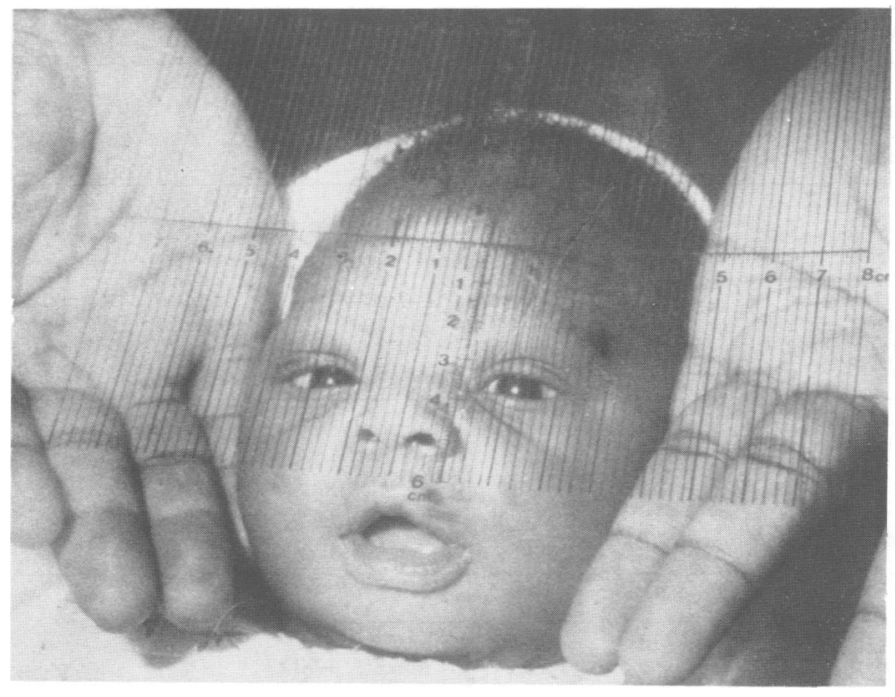

The measuring transparency, held horizontally, close to the infant's face.

to the infant's face with the baby facing upwards (figure). The head was held between the observer's hands as in the figure. The inner canthal distance (IC) was taken as the distance between the inner canthi. The medial canthus is the point where the upper lid meets the lower lid in the medial angle of the eye with the eyes at rest.

The canthal distances were measured by looking directly through the film at the landmarks beneath and reading them off. The outer canthal (OC) distance was taken as the distance between the lateral margins of the palpebral fissures with the eyes at rest.

The palpebral fissure length (PFL) was also calculated as follows:

$$
\mathrm{PFL}=\frac{\mathrm{Oc}-\mathrm{Ic}}{2}
$$

The canthal index (CI), defined as the ratio of the inner canthal distance to the outer canthal distance percent, was calculated as follows:
Canthal index $(\mathrm{CI})=\frac{100 \times \text { inner } \text { canthal distance }}{\text { Outer canthal distance }}$

The occipitofrontal circumference (OFC) was measured with a flexible Rabone Chesterman Silverflex Steel Tape (Holtain Ltd, Crymych, Wales). The tape was applied around the head at the largest circumference, the relevant landmarks being the superciliary margins in front, parietal eminences laterally, and occipital protuberance posteriorly.

The occipitofrontal circumference (OFC)/interorbital index, defined as the ratio of the inner canthal distance to the head circumference percent, was derived as follows:

$$
\mathrm{OFC} / \mathrm{IC} \text { index }=\frac{\mathrm{IC} \times 100}{\mathrm{OFC}} .
$$

\section{Results}

Tables 1 to 5 give the detailed results of the facial measurements.

\begin{tabular}{|c|c|c|c|c|c|c|c|c|c|c|}
\hline \multirow[b]{2}{*}{ Parameter } & \multicolumn{4}{|c|}{ Cardiff } & \multicolumn{3}{|c|}{ Ibadan } & \multirow[b]{2}{*}{$1 \mathrm{SD}$} & \multirow[b]{2}{*}{$t$} & \multirow[b]{2}{*}{$\mathbf{p}$} \\
\hline & $\begin{array}{l}\text { No of } \\
\text { cases }\end{array}$ & Range & Mean & $1 \mathrm{SD}$ & $\begin{array}{l}\text { No of } \\
\text { cases }\end{array}$ & Range & Mean & & & \\
\hline $\begin{array}{l}\text { Outer canthal } \\
\text { distance }(\mathrm{cm}) \\
\text { Inner canthal }\end{array}$ & 252 & $5 \cdot 0-7 \cdot 4$ & $6 \cdot 3$ & 0.4 & 256 & $5 \cdot 4-8 \cdot 0$ & $6 \cdot 8$ & 0.4 & $14 \cdot 086$ & 0.001 \\
\hline $\begin{array}{l}\text { distance (cm) } \\
\text { PFL (cm) } \\
\text { Canthal index (\%) } \\
\text { OFC (cm) } \\
\text { OFC-IC index }(\%)\end{array}$ & $\begin{array}{l}252 \\
252 \\
252 \\
252 \\
252\end{array}$ & $\begin{array}{c}1 \cdot 1-2 \cdot 5 \\
1 \cdot 6-2 \cdot 6 \\
22 \cdot 9-40 \cdot 3 \\
30 \cdot 9-39 \cdot 2 \\
5 \cdot 3-7 \cdot 1\end{array}$ & $\begin{array}{r}2 \cdot 2 \\
2 \cdot 0 \\
35 \cdot 0 \\
35 \cdot 1 \\
6 \cdot 2\end{array}$ & $\begin{array}{l}0 \cdot 2 \\
0 \cdot 2 \\
2 \cdot 4 \\
1 \cdot 4 \\
0 \cdot 4\end{array}$ & $\begin{array}{l}256 \\
256 \\
256 \\
256 \\
256\end{array}$ & $\begin{array}{c}1 \cdot 8-2 \cdot 6 \\
1 \cdot 7-4 \cdot 3 \\
23 \cdot 0-37 \cdot 5 \\
30 \cdot 0-38 \cdot 0 \\
5 \cdot 3-7 \cdot 6\end{array}$ & $\begin{array}{r}2 \cdot 2 \\
2 \cdot 3 \\
32 \cdot 0 \\
34 \cdot 6 \\
6 \cdot 3\end{array}$ & $\begin{array}{l}0 \cdot 2 \\
0 \cdot 2 \\
2 \cdot 8 \\
1 \cdot 2 \\
0 \cdot 4\end{array}$ & $\begin{array}{r}0 \cdot 000 \\
16 \cdot 904 \\
12 \cdot 957 \\
2 \cdot 817 \\
2 \cdot 817\end{array}$ & $\begin{array}{l}* \\
\mathbf{0 . 0 0 1} \\
\mathbf{0 . 0 0 1} \\
\mathbf{0 . 0 0 5} \\
\mathbf{0 . 0 1}\end{array}$ \\
\hline
\end{tabular}

Table I Range, means, and standard deviations of facial measurement, all sexes combined, Cardiff and Ibadan compared.

$t=$ Student's $t$ test. Not significant. 
Table 2 Facial measurement values, the sexes compared, in Cardiff white newborns.

\begin{tabular}{|c|c|c|c|c|c|c|c|c|c|c|}
\hline \multirow[b]{2}{*}{ Parameter } & \multicolumn{4}{|c|}{ Male } & \multicolumn{3}{|c|}{ Female } & \multirow[b]{2}{*}{$1 \mathrm{SD}$} & \multirow[b]{2}{*}{$t$} & \multirow[b]{2}{*}{$\mathbf{p}$} \\
\hline & $\begin{array}{l}\text { No of } \\
\text { cases }\end{array}$ & Range & Mean & $1 \mathrm{SD}$ & $\begin{array}{l}\text { No of } \\
\text { cases }\end{array}$ & Range & Mean & & & \\
\hline $\begin{array}{l}\text { Outer canthal } \\
\text { distance }(\mathrm{cm})\end{array}$ & 125 & $5 \cdot 3-7 \cdot 4$ & $6 \cdot 3$ & 0.4 & 127 & $5 \cdot 0-7 \cdot 4$ & $6 \cdot 2$ & 0.4 & 1.984 & 0.05 \\
\hline $\begin{array}{l}\text { Inner canthal } \\
\text { distance (cm) } \\
\text { PFL (cm) } \\
\text { Canthal index (\%) } \\
\text { OFC (cm) } \\
\text { OFC-IC index (\%) }\end{array}$ & $\begin{array}{l}125 \\
125 \\
125 \\
125 \\
125\end{array}$ & $\begin{array}{c}1 \cdot 8-2 \cdot 5 \\
1 \cdot 7-2 \cdot 6 \\
28 \cdot 1-40 \cdot 0 \\
31 \cdot 5-38 \cdot 3 \\
5 \cdot 3-7 \cdot 1\end{array}$ & $\begin{array}{r}2 \cdot 2 \\
2 \cdot 1 \\
35 \cdot 0 \\
35 \cdot 5 \\
6 \cdot 2\end{array}$ & $\begin{array}{l}0 \cdot 1 \\
0 \cdot 2 \\
2 \cdot 1 \\
1 \cdot 4 \\
0 \cdot 4\end{array}$ & $\begin{array}{l}127 \\
127 \\
127 \\
127 \\
127\end{array}$ & $\begin{array}{c}1 \cdot 1-2 \cdot 5 \\
1 \cdot 6-2 \cdot 6 \\
22 \cdot 9-40 \cdot 3 \\
30 \cdot 8-39 \cdot 2 \\
5 \cdot 4-7 \cdot 1\end{array}$ & $\begin{array}{r}2 \cdot 2 \\
2 \cdot 0 \\
34 \cdot 8 \\
34 \cdot 8 \\
6 \cdot 8\end{array}$ & $\begin{array}{l}0 \cdot 2 \\
0 \cdot 2 \\
2 \cdot 6 \\
1 \cdot 3 \\
0 \cdot 4\end{array}$ & $\begin{array}{l}0.000 \\
3.969 \\
0 \cdot 671 \\
4 \cdot 114 \\
1.984\end{array}$ & $\begin{array}{l}{ }^{*} \\
0.001 \\
0.05^{*} \\
0.001 \\
0.05\end{array}$ \\
\hline
\end{tabular}

${ }^{*}$ Not significant.

Table 3 Facial measurement values, the sexes compared, in Ibadan black newborns.

\begin{tabular}{|c|c|c|c|c|c|c|c|c|c|c|}
\hline \multirow[b]{2}{*}{ Parameter } & \multicolumn{4}{|c|}{ Male } & \multicolumn{3}{|c|}{ Female } & \multirow[b]{2}{*}{$1 \mathrm{SD}$} & \multirow[b]{2}{*}{$t$} & \multirow[b]{2}{*}{$\mathbf{p}$} \\
\hline & $\begin{array}{l}\text { No of } \\
\text { cases }\end{array}$ & Range & Mean & 1 SD & $\begin{array}{l}\text { No of } \\
\text { cases }\end{array}$ & Range & Mean & & & \\
\hline $\begin{array}{l}\text { Outer canthal } \\
\text { distance }(\mathrm{cm})\end{array}$ & 132 & $5 \cdot 9-9 \cdot 2$ & $5 \cdot 9$ & 0.4 & 124 & $5 \cdot 4-8 \cdot 0$ & $6 \cdot 8$ & 0.4 & 1.999 & 0.05 \\
\hline $\begin{array}{l}\text { distance (cm) } \\
\text { PFL (cm) } \\
\text { Canthal index (\%) } \\
\text { OFC (cm) } \\
\text { OFC-IC index (\%) }\end{array}$ & $\begin{array}{l}132 \\
132 \\
132 \\
132 \\
132\end{array}$ & $\begin{array}{c}1 \cdot 8-2 \cdot 6 \\
2 \cdot 0-2 \cdot 9 \\
27 \cdot 5-37 \cdot 5 \\
31 \cdot 0-38 \cdot 0 \\
5 \cdot 3-7 \cdot 4\end{array}$ & $\begin{array}{r}2 \cdot 2 \\
2 \cdot 3 \\
32 \cdot 0 \\
34 \cdot 9 \\
6 \cdot 3\end{array}$ & $\begin{array}{l}0 \cdot 2 \\
0 \cdot 2 \\
2 \cdot 1 \\
1 \cdot 2 \\
0 \cdot 4\end{array}$ & $\begin{array}{l}124 \\
124 \\
124 \\
124 \\
124\end{array}$ & $\begin{array}{c}1 \cdot 8-2 \cdot 6 \\
1 \cdot 7-4 \cdot 3 \\
33 \cdot 0-37 \cdot 0 \\
30 \cdot 0-36 \cdot 5 \\
5 \cdot 5-7 \cdot 6\end{array}$ & $\begin{array}{r}2 \cdot 2 \\
2 \cdot 3 \\
31 \cdot 7 \\
34 \cdot 2 \\
6 \cdot 3\end{array}$ & $\begin{array}{l}0 \cdot 2 \\
0 \cdot 3 \\
3 \cdot 3 \\
1 \cdot 2 \\
0 \cdot 4\end{array}$ & $\begin{array}{l}0.000 \\
0.000 \\
0.878 \\
4.664 \\
0.000\end{array}$ & $\begin{array}{c}* \\
* \\
0 \cdot 5^{*} \\
0 \cdot 001 \\
*\end{array}$ \\
\hline
\end{tabular}

${ }^{*}$ Not significant.

Table 4 Facial measurement values compared between Ibadan and Cardiff newborn males.

\begin{tabular}{|c|c|c|c|c|c|c|c|c|c|c|}
\hline \multirow[b]{2}{*}{ Parameter } & \multicolumn{4}{|c|}{ Cardiff } & \multicolumn{3}{|c|}{ Ibadan } & \multirow[b]{2}{*}{$1 \mathrm{SD}$} & \multirow[b]{2}{*}{$t$} & \multirow[b]{2}{*}{ p } \\
\hline & $\begin{array}{l}\text { No of } \\
\text { cases }\end{array}$ & Range & Mean & $1 \mathrm{SD}$ & $\begin{array}{l}\text { No of } \\
\text { cases }\end{array}$ & Range & Mean & & & \\
\hline $\begin{array}{l}\text { Outer canthal } \\
\text { distance }(\mathrm{cm}) \\
\text { Inner canthal }\end{array}$ & 125 & $5 \cdot 3-7 \cdot 4$ & $6 \cdot 3$ & 0.4 & 132 & $5 \cdot 9-9 \cdot 2$ & 6.9 & 0.4 & $12 \cdot 019$ & 0.001 \\
\hline $\begin{array}{l}\text { distance }(\mathrm{cm}) \\
\text { PFL }(\mathrm{cm}) \\
\text { Canthal index (\%) } \\
\text { OFC (cm) } \\
\text { OFC-IC index (\%) }\end{array}$ & $\begin{array}{l}125 \\
125 \\
125 \\
125 \\
125\end{array}$ & $\begin{array}{c}1 \cdot 8-2 \cdot 5 \\
1 \cdot 7-2 \cdot 6 \\
28 \cdot 1-40 \cdot 0 \\
31 \cdot 5-38 \cdot 5 \\
5 \cdot 3-7 \cdot 1\end{array}$ & $\begin{array}{r}2 \cdot 2 \\
2 \cdot 1 \\
35 \cdot 0 \\
35 \cdot 5 \\
6 \cdot 2\end{array}$ & $\begin{array}{l}0 \cdot 1 \\
0 \cdot 2 \\
2 \cdot 1 \\
1 \cdot 4 \\
0 \cdot 4\end{array}$ & $\begin{array}{l}132 \\
132 \\
132 \\
132 \\
132\end{array}$ & $\begin{array}{c}1 \cdot 8-2 \cdot 6 \\
2 \cdot 0-2 \cdot 9 \\
27 \cdot 5-37 \cdot 5 \\
31 \cdot 0-38 \cdot 0 \\
5 \cdot 3-7 \cdot 4\end{array}$ & $\begin{array}{r}2 \cdot 2 \\
2 \cdot 3 \\
32 \cdot 0 \\
34 \cdot 9 \\
6 \cdot 3\end{array}$ & $\begin{array}{l}0 \cdot 2 \\
0 \cdot 2 \\
2 \cdot 1 \\
1 \cdot 2 \\
0 \cdot 4\end{array}$ & $\begin{array}{r}0 \cdot 000 \\
8 \cdot 013 \\
11 \cdot 447 \\
3.695 \\
2.003\end{array}$ & $\begin{array}{c}* \\
0.001 \\
0.001 \\
0.001 \\
0.005\end{array}$ \\
\hline
\end{tabular}

*Not significant.

Table 5 Facial measurement values compared between Ibadan and Cardiff newborn females.

\begin{tabular}{|c|c|c|c|c|c|c|c|c|c|c|}
\hline \multirow[b]{2}{*}{ Parameter } & \multicolumn{4}{|c|}{ Cardiff } & \multicolumn{3}{|c|}{ Ibadan } & \multirow[b]{2}{*}{$1 \mathrm{SD}$} & \multirow[b]{2}{*}{$t$} & \multirow[b]{2}{*}{$\mathbf{p}$} \\
\hline & $\begin{array}{l}\text { No of } \\
\text { cases }\end{array}$ & Range & Mean & $1 \mathrm{SD}$ & $\begin{array}{l}\text { No of } \\
\text { cases }\end{array}$ & Range & Mean & & & \\
\hline $\begin{array}{l}\text { Outer canthal } \\
\text { distance }(\mathrm{cm})\end{array}$ & 127 & $5 \cdot 0-7 \cdot 4$ & $6 \cdot 2$ & 0.4 & 124 & $5 \cdot 4-8 \cdot 0$ & $6 \cdot 8$ & 0.4 & $11 \cdot 881$ & 0.001 \\
\hline $\begin{array}{l}\text { Inner canthal } \\
\text { distance (cm) } \\
\text { PFL (cm) } \\
\text { Canthal index (\%) } \\
\text { OFC (cm) } \\
\text { OFC-IC index (\%) }\end{array}$ & $\begin{array}{l}127 \\
127 \\
127 \\
127 \\
127\end{array}$ & $\begin{array}{c}1 \cdot 1-2 \cdot 5 \\
1 \cdot 6-2 \cdot 6 \\
22 \cdot 9-40 \cdot 3 \\
30 \cdot 8-39 \cdot 2 \\
5 \cdot 4-7 \cdot 1\end{array}$ & $\begin{array}{r}2 \cdot 2 \\
2 \cdot 0 \\
34 \cdot 8 \\
34 \cdot 8 \\
6 \cdot 3\end{array}$ & $\begin{array}{l}0.2 \\
0.2 \\
2.6 \\
1.3 \\
0.4\end{array}$ & $\begin{array}{l}124 \\
124 \\
124 \\
124 \\
124\end{array}$ & $\begin{array}{c}1 \cdot 8-2 \cdot 6 \\
1 \cdot 7-4 \cdot 3 \\
33 \cdot 0-32 \cdot 0 \\
30 \cdot 0-36 \cdot 5 \\
5 \cdot 5-7 \cdot 6\end{array}$ & $\begin{array}{r}2 \cdot 2 \\
2 \cdot 3 \\
31 \cdot 7 \\
34 \cdot 2 \\
6 \cdot 3\end{array}$ & $\begin{array}{l}0 \cdot 2 \\
0 \cdot 3 \\
3 \cdot 3 \\
1 \cdot 2 \\
0 \cdot 4\end{array}$ & $\begin{array}{l}0 \cdot 000 \\
9 \cdot 342 \\
8 \cdot 227 \\
3 \cdot 797 \\
0 \cdot 000\end{array}$ & $\begin{array}{c}* \\
0.001 \\
0.001 \\
0.001 \\
*\end{array}$ \\
\hline
\end{tabular}

${ }^{*}$ Not significant. 
The outer canthal distance for white newborns in Cardiff ranged between $5 \cdot 0$ and $7 \cdot 4 \mathrm{~cm}$ (mean 6.3 (SD $0.4 \mathrm{~cm})$ ) while the Ibadan newborns ranged between 5.4 and $8.0 \mathrm{~cm}$ (mean 6.8 (SD $0.4 \mathrm{~cm}$ ), p $<0.001$ ). The inner canthal distance mean value was the same in the two groups $(2.2(S D 0.2 \mathrm{~cm}))$. There was no variation in value with respect to sex either.

The palpebral fissure length ranged from 1.6 to 2.6 $\mathrm{cm}$ (mean $2.0($ SD $0.2 \mathrm{~cm})$ ) in the Cardiff group and varied from 1.7 to $4.3 \mathrm{~cm}$ (mean 2.3 (SD $0.2 \mathrm{~cm}$ )) in the Ibadan group. The higher Ibadan mean value was statistically significant $(\mathrm{p}<0.001)$ and within the Ibadan group there was no sex difference as they both had the same mean value $(2.3($ SD $0.3 \mathrm{~cm}))$.

The occipitofrontal circumference for the Cardiff group (mean 35.1 (SD $1.4 \mathrm{~cm})$ ) was significantly larger than the Ibadan group (mean 34.6 (SD $1 \cdot 2$ $\mathrm{cm}), \mathrm{p}<0.001$ ).

The occipitofrontal circumference/inner canthal index values for Cardiff ranged between $5 \cdot 3$ and $7 \cdot 1 \%$ (mean 6.2 (SD 0.4)) and the Ibadan values ranged between $5 \cdot 3$ and $7 \cdot 6 \%$ (mean $6 \cdot 3$ (SD $0 \cdot 4 \%$ )).

\section{Discussion}

The facial values obtained in this series allowed for a truly comparative study as the same instruments were used by one person on all the subjects. This minimised any observer error. Similar outer canthal readings obtained for newborns in the series of Feingold and Gellis $^{3}$ were also obtained for newborns in Cardiff (mean $6.3 \mathrm{~cm}$ ). Ejiwunmi et $a l^{7}$ obtained a slightly lower value $(6.7 \mathrm{~cm}(S D \mathrm{0.4}))$ for Nigerian newborns than in this series, though their value agreed quite well with readings (mean $6.9(\mathrm{SD} 0.4 \mathrm{~cm}$ )) for the Ibadan babies. The Negroid neonate is hyperteloric with respect to the outer canthal distance, but not to the inner canthal distance, as confirmed by several authors (table 6).
The palpebral fissure lengths in Negroid newborns were significantly wider than in their Caucasoid counterparts. This must be taken into consideration in checking for congenital glaucoma in newborns of African descent. Jones et $a l^{10}$ remarked that the palpebral fissure length appears to relate to eye size, and because the eye is a neural organ PFL indirectly reflects that aspect of neural growth. Geber and Dean ${ }^{11}$ observed advanced psychomotor development of the African at birth.

Could this have been reflected in the observed differences in palpebral fissure length? It is important also to follow the rate of growth of the PFL to see if there is a reduction in growth velocity, as the observed precocity of the African has been noted to fall off, especially around weaning time. This is currently being studied.

Measurements of the face are very important in describing accurately the syndromes that have facial involvement. The eyes may be really wide apart as in Apert's, Crouzon's, and Robinson's syndromes. Conversely, the eyes may be unusually close together as in holoprosencephaly anomalad, trisomy 13, Williams', and the Meckel-Gruber syndromes. These syndromes are easily recognised, but the abnormalities are not so clear in unaffected family members who may only manifest partial features. Subjecting such questionable traits to measurement would help in risk calculation and in genetic counselling.

The use of a single measurement standard for all, as advocated by some workers, cannot be supported in the light of this work. The only exception is perhaps in the measurement of the inner canthal distance. To subject all to a single standard would either underdiagnose or overdiagnose ocular hypertelorism depending on the races involved.

It is advocated that these readings should be continued through to adulthood and in disease conditions.

Table 6 Means and standard deviations of facial measurements (from several authors)

\begin{tabular}{|c|c|c|c|c|c|c|c|c|}
\hline \multirow[b]{2}{*}{ Authors } & \multicolumn{2}{|c|}{$\begin{array}{l}\text { Outer canthal } \\
\text { distance }(\mathrm{cm})\end{array}$} & \multicolumn{2}{|c|}{$\begin{array}{l}\text { Inner canthal } \\
\text { distance }(\mathrm{cm})\end{array}$} & \multicolumn{2}{|c|}{$\begin{array}{c}\text { Canthal } \\
\text { index }(\%)\end{array}$} & \multicolumn{2}{|c|}{$\begin{array}{c}\text { Palpebral } \\
\text { fissure length }\end{array}$} \\
\hline & $\mathbf{M}$ & $\mathbf{F}$ & $\mathbf{M}$ & $\mathbf{F}$ & $M$ & $\mathbf{F}$ & $\mathbf{M}$ & $\mathbf{F}$ \\
\hline $\begin{array}{l}\text { Ejiwunmi et } a l^{7} \\
\text { (Lagos) }\end{array}$ & $\begin{array}{l}6 \cdot 7 \\
(0 \cdot 4)\end{array}$ & $\begin{array}{l}6 \cdot 6 \\
(0 \cdot 7)\end{array}$ & $\begin{array}{l}2 \cdot 0 \\
(0 \cdot 2)\end{array}$ & $\begin{array}{l}2 \cdot 0 \\
(0 \cdot 2)\end{array}$ & $29 \cdot 9$ & $30 \cdot 3$ & * & : \\
\hline $\begin{array}{l}\text { This study } \\
\text { (Ibadan) } \\
\text { This study } \\
\text { (Cardiff) } \\
\text { Feingold and Gellis }{ }^{3} \\
\text { (Boston) }\end{array}$ & $\begin{array}{l}6 \cdot 9 \\
(0 \cdot 4) \\
6 \cdot 3 \\
(0 \cdot 4) \\
6 \cdot 3\end{array}$ & $\begin{array}{l}6 \cdot 8 \\
0 \cdot 4 \\
6 \cdot 2 \\
(0 \cdot 4)\end{array}$ & $\begin{array}{l}2 \cdot 2 \\
(0 \cdot 2) \\
2 \cdot 2 \\
(0 \cdot 1) \\
2 \cdot 0\end{array}$ & $\begin{array}{l}2 \cdot 2 \\
(0 \cdot 2) \\
2 \cdot 2 \\
(0 \cdot 2)\end{array}$ & $\begin{array}{l}32 \cdot 0 \\
(2 \cdot 1) \\
35 \cdot 0 \\
(2 \cdot 1) \\
31 \cdot 7\end{array}$ & $\begin{array}{l}31 \cdot 7 \\
(3 \cdot 3) \\
34 \cdot 8 \\
(2 \cdot 6)\end{array}$ & $\begin{array}{l}2 \cdot 3 \\
(0 \cdot 2) \\
2 \cdot 1 \\
(0 \cdot 2) \\
? \cdot\end{array}$ & $\begin{array}{l}2 \cdot 3 \\
(0 \cdot 3) \\
2 \cdot 0 \\
(0 \cdot 2) \\
*\end{array}$ \\
\hline $\begin{array}{l}\text { Ogunye et } a l^{t} \\
\text { (Ife) }\end{array}$ & $*$ & * & $\begin{array}{l}1 \cdot 9 \\
0.4\end{array}$ & $*$ & * & * & * & * \\
\hline $\begin{array}{l}\text { Jones et al } \\
\text { (San Diego) } \\
\text { Marden } e t a l^{\times} \\
\text {(Madison) }\end{array}$ & $*$ & * & $2 \cdot 1$ & $*$ & $*$ & $*$ & $\begin{array}{l}1 \cdot 85 \\
(0 \cdot 1)\end{array}$ & * \\
\hline
\end{tabular}

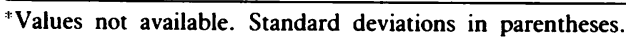


The author expresses his grateful acknowledgement to Professor K M Laurence, Institute of Medical Genetics, University of Wales College of Medicine, Cardiff, for his help and encouragement, to Jean Dunscombe for secretarial assistance, and to $\mathrm{Mr} \mathrm{A}$ Shaw of the Department of Medical Illustration of the University of Wales College of Medicine for helping to produce the transparency for the facial measurements. Many thanks also go to the medical and nursing teams of the University College Hospital and Adeoyo State Hospital, Ibadan, and University Hospital of Wales and St David's Hospital, Cardiff.

1 Feingold $M$. Proper measurements in physical diagnosis. Am $\mathcal{F}$ Dis Child 1983;137:828.
2 Laestadius ND. Normal inner canthal and outer orbital dimensions. F Pediatr 1969;74:465-71

3 Feingold $M$, Gellis SS. Syndrome identification and consultation. Am 7 Dis Child 1971;121:82-3.

4 Lucas WP, Pryor HB. Range and standard of deviations of certain physical measurements in healthy children. $\mathcal{f}$ Pediatr 1935;6:533-45.

5 Juberg RC, Sholte FG, Touchstone WJ. Normal values for intercanthal distances of 5 to 11 year old American blacks. Pediatrics 1975;55:431-6.

6 Ogunye O, Ikeji MO, Adeodu O. Craniofacial dimensions in the African neonate. Niger $\mathcal{J}$ Paediatr 1982;9:21.

7 Ejiwunmi AB, Okanlawon OA, Ojo OO. Interpupillary and inter nipple distances and ear lengths in Nigerian newborns. Ann Trop Paediatr 1984;4:103-6.

8 Marden PM, Smith DW, McDonald MJ. Congenital abnormalities in the newborn infant including minor variations. 7 Pediatr 1964;64:357-71.

9 Dubowitz LMS, Dubowitz V, Goldberg C. Clinical assessment of gestational age in the newborn infant. $\mathcal{F}$ Pediatr 1970;77:1-10.

10 Jones KL, Hanson JW, Smith DW. Palpebral fissure length in newborn infants. $\mathcal{F}$ Pediatr 1978;92:787-8.

11 Geber M, Dean RFA. Gesell tests on African children. Pediatrics 1957;20:1055-65. 